\title{
Consenso sobre riesgo de complicaciones infecciosas en pacientes usuarios de medicamentos biológicos seleccionados. Parte I
}

\begin{abstract}
Inés Cerón', Pilar Gambra², Cecilia Vizcaya33, Marcela Ferres³, Teresa Bidart4, Tania López1, M. Paz Acuña 5,6, Ana M. Álvarez', Marcela Zubieta ${ }^{8}$, Marcela Rabello ${ }^{9}$, Mirentxu Iruretagoyena ${ }^{10}$ y Ricardo Rabagliati ${ }^{1}$
\end{abstract}

'Departamento de Enfermedades Infecciosas del Adulto. Escuela de Medicina. Pontifica Universidad Católica de Chile, Santiago, Chile. Unidad de Medicina Interna e Infectología. Clínica Santa María. Santiago, Chile. ${ }^{3}$ Departamento de Enfermedades Infecciosas del Niño. Escuela de Medicina. Pontificia Universidad Católica de Chile. Santiago, Chile. ${ }^{4}$ Unidad de Infectología. Clínica Santa María, Santiago, Chile.

${ }^{5}$ Unidad de Infectología. Complejo Asistencial Dr. Sótero

del Río, Santiago, Chile.

${ }^{6}$ Unidad de Infectología. Hospital

Metropolitano de La Florida,

Santiago, Chile

${ }^{7}$ Servicio de Pediatría. Unidad de

Infectologia, Hospital San Juan de

Dios, Santiago, Chile.

Unidad de Oncología Hospita

Exequiel González Cortés.

Santiago, Chile.

'Unidad de Infectología. Hospital

Dr. Luis Calvo Mackenna. Santiago, Chile.

${ }^{10}$ Departamento de Reumatología e Inmunología Clínica. Escuela de

Medicina. Pontificia Universidad Católica de Chile. Santiago, Chile.

Los miembros del panel declaran no presentar conflictos de interés

para el desarrollo de esta guía

clínica.

El presente trabajo no recibió

financiamiento alguno.

Recibido: 21 de junio de 2019.

Correspondencia a: Inés Cerón imceron@med.puc.cl

\section{Consensus of infectious complications in patients treated with selected biological therapies: first Part}

The use of biological therapies has meant a great improvement in the management of several conditions like autoimmune, neoplastic or others diseases. Although its use has implied significant improvements in the prognosis of these diseases, it is not exempt from complications: infectious diseases as one of them. The objective of this consensus was to evaluate, from an infectious viewpoint, the safeness of the most frequently used biological therapies and give recommendations for the prevention of infections in patients treated with these drugs. These recommendations were based on the highest quality evidence available for the selected biologics. The consensus counts of two manuscripts. This first part details the risks of developing infectious complications depending on the type of biological used for a certain pathology. This evaluation included a broad search in MEDLINE and Epistemonikos of systematic reviews and meta-analyzes of controlled clinical trials and case- control examining post-treatment infections with anti-TNF alpha, anti-CD20, anti-CD52, CTLA4-Ig and anti-integrins. The research was complemented by a review of: multicentre cohorts of biological users, the MMWR of the CDC, Atlanta, U.S.A., and national registers and scientific societies in which infectious complications derived from the use of biological therapies were mentioned.

Keywords: Infection risk; biological drugs; immunocompromised host.

Palabras clave: Riesgo de infección; medicamentos biológicos; hospedero inmunocomprometido.

\section{Introducción}

L a terapia biológica consiste en el uso de anticuerpos monoclonales o proteínas de fusión para modular la función del sistema inmune. Están dirigidos a interferir citoquinas, receptores de linfocitos $\mathrm{T}$ o B y moléculas de adhesión, que han demostrado ser importantes en la patogenia de distintas enfermedades autoinmunes. El bloqueo específico de estas vías de señalización ha contribuido en forma muy significativa en el tratamiento de estas patologías ${ }^{1}$.

El uso de estos anticuerpos monoclonales tiene ventajas al compararlos con las terapias inmunosupresoras más clásicas, como por ejemplo los corticosteroides ${ }^{2}$. Pueden interferir específicamente con vías de activación o regulación de la respuesta inmune y tienen una alta afinidad y especificidad. Brevemente se mencionarán algunas de las alternativas que existen actualmente en uso y sus mecanismos de acción.

Dentro de las terapias biológicas que interfieren con vías de señalización de citoquinas están los anticuerpos anti-factor de necrosis tumoral alfa (anti-FNT alfa).
Concentraciones plasmáticas altas de esta citoquina han sido descritas en enfermedades autoinmunes como artritis reumatoide (AR) y enfermedad de Crohn, entre otras ${ }^{3}$ y su inhibición como parte de su tratamiento ha demostrado resultados favorables ${ }^{4,5}$

Este grupo incluye terapias tales como:

- Infliximab: anticuerpo quimérico (origen humano/ murino)

- Etanercept: proteína de fusión del receptor soluble de FNT alfa asociado a una región Fc IgG1.

- Adalimumab y golimumab: anticuerpos humanos.

- Certolizumab: fragmento Fab PEG (fragmento de unión a antígeno $=$ Fab por sus siglas en inglés, unido a polietilenglicol) de un anticuerpo humanizado.

El bloqueo de otra citoquina como IL-6 también ha demostrado utilidad en el tratamiento de enfermedades autoinmunes tales como artritis reumatoide (AR). Tocilizumab es un anticuerpo monoclonal humano IgG anti receptor de $I L-6$; actualmente es una alternativa de tratamiento para esta enfermedad ${ }^{6}$. En psoriasis, el bloqueo de la vía IL-12 e IL-23 con el anticuerpo monoclonal 
humano ustekinumab y el bloqueo de IL-17A mediante el anticuerpo monoclonal humano $\mathrm{IgG1}$, secukinumab, han demostrado gran utilidad en su tratamiento ${ }^{7}$.

Los anticuerpos monoclonales también pueden interferir en procesos de activación del linfocito T, como por ejemplo abatacept, que consiste en una proteína de fusión del receptor CTLA-4 unido a una región Fc. CTLA-4 es un receptor que regula la activación del linfocito $\mathrm{T}$, disminuyendo la inflamación en enfermedades autoinmunes como $\mathrm{AR}^{8}$.

También se encuentran en uso otros modificadores de la respuesta biológica tales como rituximab, un anticuerpo monoclonal anti linfocitos B (anti-CD-20). Este anticuerpo monoclonal de origen murino reconoce un receptor de superficie de linfocitos $\mathrm{B}$, eliminando linfocitos pre-B y maduros. La destrucción ocurre por varios mecanismos: lisis directa, citotoxicidad inducida por complemento y citotoxicidad inducida por células. Se utiliza en el tratamiento del linfoma y de varias enfermedades autoinmunes ${ }^{9}$.

Otra alternativa que ha demostrado ser beneficiosa en el tratamiento de enfermedades autoinmunes como esclerosis múltiple, es la inhibición de moléculas de adhesión mediante anticuerpos monoclonales. Un ejemplo es natalizumab, un anticuerpo humanizado anti-integrina alfa 4. Al bloquear esta molécula de adhesión, se interfiere con la capacidad de células del sistema inmune de migrar hacia el SNC, disminuyendo la inflamación ${ }^{10}$. Otro fármaco, alemtuzumab, es un anticuerpo anti-CD52, también efectivo en reducir recaídas por esta enfermedad ${ }^{11}$, además de otras tales como leucemia linfática crónica o linfoma no-Hodgkin ${ }^{12}$. Dado que los CD52 son antígenos de superficie celular presentes en grandes cantidades en los linfocitos $\mathrm{T}$ y $\mathrm{B}$ y, en menores cantidades, en células natural killer, monocitos y macrófagos, la unión de alemtuzumab a este receptor desencadena citólisis celular dependiente de anticuerpos y también mediada por complemento ${ }^{13}$.

Si bien la eficacia clínica de estas terapias biológicas ha sido claramente demostrada en varias patologías de origen autoinmune, neoplásico u otros, es sabido también que su uso puede conllevar riesgo de efectos adversos de diversa magnitud, dependiendo del tipo de terapia utilizada. Dentro de los principales efectos adversos descritos destacan aquellos de la esfera infecciosa, reacciones anafilácticas y neoplasias, entre otros.

El objetivo de este consenso fue, en esta primera parte, revisar el perfil de seguridad, desde la mirada infectológica, de las terapias biológicas de uso más frecuente y, en la segunda parte, entregar recomendaciones para la prevención de infecciones en pacientes tratados con ellas, basándose en la evidencia de mayor calidad disponible para aquellos medicamentos biológicos seleccionados.

\section{Metodología}

\section{Génesis del proyecto}

Durante el segundo semestre de 2016, a raíz del creciente uso de terapias biológicas y la poca claridad respecto a los reales riesgos de infección por utilización de los diferentes agentes biológicos, al interior del Comité de Infecciones en Pacientes Inmunocomprometidos de Sociedad Chilena de Infectología, surgió la necesidad de revisar las principales complicaciones infecciosas asociadas al uso de este tipo de terapias, a fin de poder desarrollar una guía con recomendaciones de medidas preventivas destinadas a minimizar el riesgo de infección según el tipo de terapia biológica aplicada.

La intención fue generar información de referencia, tanto para médicos de aquellas especialidades de adultos y niños, que indican este tipo de terapias, dentro de los que se incluyen reumatólogos, gastroenterólogos, dermatólogos, hemato-oncólogos, entre otros, como también a especialistas infectólogos.

\section{Composición del panel}

El panel se constituyó con la presencia de nueve expertos infectólogos, tanto de adultos (IC, PG, RR, TB, TL, LD) como pediátricos (CV, MF, AMA, MZ, MR) y se solicitó la asesoría de un experto en medicina basada en la evidencia (MPA) para la optimización de la búsqueda, selección y análisis de la bibliografía revisada. Además, se contó con la asesoría de un inmunólogo (MI) para el correcto entendimiento de los mecanismos de acción de los medicamentos biológicos revisados.

\section{Selección de medicamentos biológicos incluidos}

El número de nuevas terapias biológicas aumenta año a año, incorporándose de manera creciente dentro del arsenal terapéutico disponible de diferentes patologías oncológicas, inflamatorias y autoinmunes. Algunas terapias son de amplio uso para diversas patologías y por diferentes especialidades; mientras que otros son de uso muy específico para patologías de baja frecuencia. El grupo de trabajo decidió incorporar sólo aquellos biológicos de mayor uso en la actualidad, pertenecientes a la familia de: inhibidores de factores de necrosis tumoral alfa (antiFNT alfa), inhibidores de la co-estimulación de células T (CTLA-4), depletores de linfocitos B (anti-CD20), inhibidores de moléculas de adhesión (anti-integrinas) y anticuerpo monoclonal anti-CD52. La Tabla 1 resume los biológicos incluidos en este consenso y las patologías específicas en las que se determinó su riesgo de desarrollo de infecciones.

\section{Revisión de evidencia científica}

Se realizó búsqueda en MEDLINE (National Library of Medicine, Bethesda, MD) desde 1970 hasta agosto 


\begin{tabular}{|c|c|c|}
\hline Familia de biológico & Nombre de biológicos & Indicación revisada \\
\hline \multirow[t]{5}{*}{ anti-FNT alfa } & Adalimumab & $A R$ \\
\hline & Certolizumab & \multirow{2}{*}{ Psoriasis (incluyendo artritis psoriática) } \\
\hline & Golimumab & \\
\hline & Infliximab & \multirow[t]{2}{*}{ Enfermedad inflamatoria intestinal (enfermedad de Crohn, colitis ulcerosa) } \\
\hline & Etanercept & \\
\hline Inhibidores de la co-estimulación de linfocitos T (CTLA4-Ig) & Abatacept & $A R$ \\
\hline \multirow[t]{2}{*}{ Depletores de linfocitos B (anti-CD20) } & \multirow[t]{2}{*}{ Rituximab } & Neoplasias hematológicas (linfoma) \\
\hline & & AR \\
\hline \multirow[t]{2}{*}{ Bloqueadores de moléculas de adhesión (anti-integrinas) } & Natalizumab & \multirow[t]{2}{*}{ Enfermedad inflamatoria intestinal (enfermedad de Crohn, colitis ulcerosa) } \\
\hline & Vedolizumab & \\
\hline \multirow[t]{2}{*}{ Anticuerpo monoclonal anti-CD52 } & \multirow[t]{2}{*}{ Alemtuzumab } & Linfoma no-Hodgkin \\
\hline & & Esclerosis múltiple \\
\hline
\end{tabular}

2016, sin restricción de idiomas, utilizando como filtro revisiones sistemáticas y meta-análisis de estudios clínicos controlados y caso/control que examinaban infecciones (graves, oportunistas, no graves y peri-operatorias) con posterioridad al tratamiento con anti-FNT alfa, anti-CD20, anti-CD52, CTLA4-Ig y anti-integrinas. La búsqueda fue específica para las siguientes enfermedades: cáncer, $\mathrm{AR}$, enfermedad inflamatoria intestinal, psoriasis, artritis psoriática y enfermedades inflamatorias en su conjunto. Las definiciones de infecciones de cualquier tipo, oportunistas, graves y peri-operatorias fueron las reportadas por cada autor en particular. De esta búsqueda se obtuvo 36 revisiones sistemáticas relevantes para el tema, que fueron evaluadas por el equipo, seleccionándose finalmente 12 para ser incluidas en la revisión. Paralelamente, se realizó búsqueda en la base de datos Epistemonikos utilizando como criterio de búsqueda todos aquellos artículos que mencionaran "biológicos" y "efectos adversos" en su resumen, seleccionándose aquellas revisiones que reportaron resultados de seguridad. Se obtuvo 39 revisiones sistemáticas adicionales relevantes para el tema, de las cuales se seleccionaron las seis que mejor respondían las inquietudes planteadas por el panel. Se privilegió las revisiones sistemáticas más recientes y de mejor calidad metodológica que fueran atingentes a los objetivos de la guía a desarrollar. La bibliografía de las revisiones sistemáticas seleccionadas también fue evaluada en búsqueda de referencias adicionales relevantes. Cuando se consideró necesario, se complementó la búsqueda revisando los estudios clínicos randomizados controlados (sigla en inglés $=$ RCT, para randomized controlled trials) originales; además de revisión de cohortes multicéntricas de usuarios de biológicos en los que se reportaran eventos adversos de la esfera infectológica, revisión de la publicación Morbidity and Mortality Weekly Report (MMWR) del CDC, Atlanta, E.U.A., y revisión de registros nacionales y/o de sociedades científicas en la que se hiciera mención a complicaciones infecciosas derivadas del uso de biológicos.

\section{Estructura del documento consenso}

El panel acordó estructurar la revisión y recomendaciones en dos manuscritos. El primero de éstos detalla los riesgos de desarrollar diversas complicaciones infecciosas dependiendo del tipo de medicamento biológico utilizado para determinada patología; mientras que el segundo apunta a ser una guía de práctica clínica con recomendaciones para la prevención de infecciones por bacterias, micobacterias en particular, virus, hongos y parásitos, tanto para adultos como para niños, que pudieran presentarse asociadas al uso de los biológicos estudiados. Si bien la idea original del panel era realizar estas recomendaciones basadas en evidencia de alta calidad, utilizando el sistema GRADE (Grading of Recommendations, Assessment, Development and Evaluation), la falta de información de respaldo para ciertos escenarios clínicos obligó al panel a realizar muchas de sus recomendaciones basadas en opinión de expertos. 


\section{Riesgo de infecciones según biológicos}

En términos generales, la evidencia disponible apoya la idea de un riesgo aumentado de infecciones en usuarios de terapias biológicas; aunque muchas veces los RCT, principalmente por diseño y tiempo de seguimiento, no tienen el poder suficiente para identificar específicamente las etiologías de estas infecciones. La red de meta-análisis de la Cochrane Database Syst Rev ${ }^{14}$ que incluyó 160 RCT (48.676 pacientes) y 46 estudios abiertos (11.954 pacientes) con uso de anti-FNT alfa (adalimumab, certolizumab, etanercept, golimumab, inflimixab), antagonista IL-1 (anakinra), antagonista IL-6 (tocilizumab), CTLA4-Ig (abatacept) o anti-CD20 (rituximab), para cualquier enfermedad que no incluyera pacientes con infección por VIH/SIDA, mostró un incremento significativo en el riesgo de presentar infecciones graves, definidas por necesidad de hospitalización, uso de antimicrobianos parenterales o muerte, con un Odds-ratio (OR) 1,37 (IC $95 \% ; 1,04-1,82)$ y con un número necesario para causar daño (en inglés $=$ NNH number needed to harm $)$ de 108 (IC 95\%; 50-989). Además, se observó un incremento significativo en el riesgo de presentar tuberculosis (TBC) ya fuese como debut, reactivación pulmonar, cavitada o miliar, o de cualquier órgano con un OR de 4,68 (IC 95\%; 1,18-18), con un NNH de 681 (IC 95\%; 143-14706). Al analizar las terapias biológicas por separado, sólo certolizumab y anakinra se asociaron a mayor riesgo de infecciones graves comparados con controles [OR 4,75 (IC 95\%; 1,52-18,65) con NNH de 12 (IC 95\%; 4-79) y OR 4,05, 95\% (IC 95\%; 1,22-16,84) con NNH de 14 (IC 95\%; 4-181), respectivamente.

\section{Anti-FNT alfa: infliximab, adalimumab, golimumab, certolizumab, etanercept}

Son la familia de biológicos más grande y con mayor variedad de indicaciones. Los anti-FNT alfa han demostrado eficacia en diversas patologías, tales como $\mathrm{AR}$, psoriasis, enfermedades inflamatorias intestinales (colitis ulcerosa y enfermedad de Crohn), espondilitis anquilosante, etc.

La principal indicación de los anti- FNT alfa es el tratamiento de AR refractaria a terapias convencionales. Comparado con los fármacos modificadores de AR (FMAR) tradicionales, los biológicos (anti- FNT alfa anti-CD20, CTLA4-Ig e inhibidores de IL-6) en dosis estándar y en altas dosis, incrementan el riesgo de infecciones graves OR 1,31 (IC 95\%; 1,09-1,58) y OR 1,90 (IC 95\%; 1,50-2,39), respectivamente ${ }^{15}$. Al desglosar por biológico específico, los que más riesgo presentaron fueron adalimumab (OR 1,86 [IC 95\%; 1,15-3,01]) y certolizumab (OR 3,61 [IC 95\%; 1,31-9,99]). Bongartz y cols. ${ }^{16}$, demostraron que infliximab y adalimumab estaban asociados con un incremento significativo en infecciones graves (OR 2 [IC 95\%; 1,3-3,1]), principalmente por infecciones granulomatosas (10 TBC, 1 histoplasmosis y 1 coccidiomicosis). La infección grave más frecuente fue la $\mathrm{TBC}^{17}$. Un análisis de seguridad de un registro español de pacientes con AR reportó más de 50 veces mayor riesgo de TBC (RR 53 (IC 95\%; 34,5-89) en pacientes tratados con infliximab comparados con los que no recibieron medicamentos anti- FNT alfa ${ }^{18}$. Muchos estudios observacionales indican que infliximab puede llevar a un riesgo mayor de $\mathrm{TBC}$ u otras infecciones granulomatosas, e incluso, a un brote más rápido de TBC al compararlo con etanercept ${ }^{17-19}$.

Esto queda claramente reflejado por Dixon y cols., que compararon las tasas de TBC entre 10.712 pacientes con AR tratados con anti- FNT alfa (3.295 infliximab, 3.504 adalimumab y 3.913 etanercept) y 3.232 con terapia tradicional registrados por la Sociedad Británica de Reumatología ${ }^{20}$. En este estudio, se identificaron 40 casos sólo en el grupo de anti- FNT alfa, destacando una incidencia específica por biológico de 136, 144 y 39 casos/100.000 personas-año para infliximab, adalimumab y etanercept, con tiempos medios de presentación de 5,5, 18,5 y 13,4 meses, respectivamente. De estas infecciones, $62 \%$ fueron extra-pulmonares, $44 \%$ de los cuales fueron diseminadas, y destacó que $32,5 \%$ de los casos se presentaron luego de suspender la terapia biológica. Una revisión más reciente de casos de TBC en pacientes receptores de biológicos, refuerza que el grupo de pacientes que reciben anti- FNT alfa son quienes se encuentran en mayor riesgo, sumando también aquellos que se encuentran en tratamiento con golimumab y certolizumab ${ }^{21}$. Es importante destacar que las descripciones de mayor frecuencia de infecciones por micobacterias también incluyen las no-tuberculosas. En el seguimiento de 8.418 pacientes receptores de anti- FNT alfa, se describe una tasa de 74 casos/100.000 personasaño con mayor frecuencia para adalimumab, luego infliximab y menos frecuente con etanercept $\mathrm{t}^{22}$.

El riesgo de desarrollo de infección varía un poco si los anti- FNT alfa son usados para tratamiento de psoriasis (artritis psoriática y psoriasis cutánea). La explicación para esta variación estaría en que los pacientes con psoriasis no utilizan habitualmente otros inmunosupresores o inmunomoduladores como sí ocurre en AR, lo que podría condicionar un riesgo menor de infección. En una revisión sistemática de Dommasch y cols. ${ }^{23}$, que incluyó $20 \mathrm{RCT}$ con 6.911 pacientes con psoriasis cutánea o artritis psoriática, se observó un leve incremento del riesgo global de infecciones con uso de corta duración (mediana 17,8 semanas) de anti- FNT alfa OR 1,18 (IC $95 \% ; 1,05-1,33)$, de las cuales $97 \%$ fueron infecciones no graves (principalmente infecciones respiratorias altas). No se observó incremento en el riesgo de infecciones graves OR 0,7 (IC 95\%; 0,40-1,21). 
Esta tendencia se repite en otro meta-análisis más reciente ${ }^{24}$ que incluyó $32 \mathrm{RCT}$ con 13.359 pacientes con psoriasis tratado con biológicos (anti- FNT alfa, ustekimumab y secukimumab) seguidos a las semanas 12-16 y 20-30, sin evidencias de mayor riesgo de infecciones graves. Destaca en esta publicación la inclusión de un estudio de cohorte prospectiva el que sí evidenció un riesgo significativamente mayor de infección grave al comparar adalimumab con fototerapia y/o retinoides; pero no muestra descripción sobre el tipo de infecciones presentadas.

En enfermedades inflamatorias intestinales como son la enfermedad de Crohn y colitis ulcerosa, Ford y cols. ${ }^{25}$, evidenciaron el doble de riesgo de desarrollar infección oportunista con el uso de anti-FNT alfa (adalimumab, certolizumab, golimumab e infliximab) vs placebo: OR 2,05 (IC 95\%; 1, 10-3,85) con NNH de 500 (IC 95\%; 2001.567). Las infecciones oportunistas reportadas fueron: 8 TBC, 8 infecciones por virus herpes simplex (VHS), 6 candidiasis oral o esofágica, 6 herpes zoster, 2 varicelas, 2 infecciones por citomegalovirus (CMV) o virus de Epstein Barr (VEB) y 1 infección por Nocardia. El riesgo relativo de presentar TBC no fue significativo para el mismo grupo de biológicos estudiados con un riesgo relativo (RR) de 2,52 (IC 95\%; 0,62-10,21).

La misma tendencia respecto a aumento de riesgo de infecciones oportunistas se observó en otra revisión sistemática $^{26}$, con OR 1,89 (IC 95\%; 1,15-3,12) para el conjunto de anti-FNT alfa, pero en forma individual ninguna molécula logró una diferencia significativa. En esta revisión destacó el mayor riesgo de infecciones oportunistas con el uso de anti- FNT alfa observado en enfermedad de Crohn respecto de colitis ulcerosa con OR 2,39 (IC 95\%; 1,32-4,34) vs OR 1,32 (IC 95\%; 0,64-2,72), aunque la diferencia no fue estadísticamente significativa.

\section{Inhibidores de la co-estimulación de células T (CTLA-4 Ig): abatacept}

El abatacept se ha utilizado con éxito en el tratamiento de AR. En una revisión sistemática de Strand y cols. ${ }^{27}$, no se evidenció incremento del riesgo de infecciones graves al usar abatacept con un OR 1,26 (IC 95\%; 0,75-2,11). En relación a TBC, si bien habría un riesgo incrementado comparado con placebo, éste sería menor que con otros biológicos. De los 32 casos de TBC detectados en 19 RCT, sólo $1 / 433(0,23 \%)$ fue con abatacept; mientras que, en estudios de extensión prolongada, la tasa de incidencia de TBC con abatacept fue de 60 (IC 95\%; 18,2-125,9), sin heterogeneidad $\left(\mathrm{I}^{2}=0 \%\right)$. Lo que fue bastante menor al de otros biológicos de la familia de los anti-FNT alfa, como por ejemplo, certolizumab con una tasa de incidencia de TBC de 474,2 (IC 95\%; 350- 640), sin heterogeneidad
$\left.\left(\mathrm{I}^{2}=0 \%\right)\right)$ o de infliximab con una tasa de incidencia de TBC de 347,7 (IC 95\%; 193,4- 539,2), sin heterogenei$\operatorname{dad}\left(\mathrm{I}^{2}=0 \%\right)^{28}$.

\section{Depletores de linfocitos B (anti-CD20): rituximab}

Existen bastantes publicaciones del uso de rituximab en pacientes con linfomas de curso desfavorable, demostrando aumento en la remisión de linfomas indolentes y linfomas agresivos CD20+. Dado que la inmunosupresión que produce rituximab es profunda y prolongada ( 6 a 9 meses), hay una tendencia en señalar que puede haber infecciones en los pacientes que lo utilizan, pero no hay claridad sobre si la adición de rituximab aumentaría efectivamente el riesgo de infecciones o no. Lanini y cols. $^{29}$, realizaron un meta-análisis que incluyó 17 RCT (5.259 pacientes) cuyo objetivo era determinar el riesgo de infección en pacientes con linfoma maligno tratados con rituximab, no lográndose evidenciar mayor riesgo de infección en este grupo de pacientes al compararlo con quimioterapia convencional: RR 1 (IC 95\%; 0,87-1,14). Tampoco evidenciaron incremento del riesgo de neutropenia febril: RR 1,14 (IC 95\%; 0,8-1,63). Solamente se observó un incremento significativo de granulocitopenia: RR 1,07 (IC 95\%; 1,02-1,12), p = 0,008. La evaluación del riesgo de infección por agentes etiológicos específicos es bastante escueta. Coiffer y cols. ${ }^{30}$, reportaron 9/202 $(4,5 \%)$ casos de herpes zoster en el grupo tratado con quimioterapia que incluyó rituximab vs 2/197 (1\%) de casos en el grupo tratado sólo con quimioterapia; mientras que Robak y cols. ${ }^{31}$, en un estudio que sólo consideró pacientes sin hepatitis B crónica, reportaron 5/272 (1,8\%) casos de hepatitis $\mathrm{B}$ en el grupo quimioterapia más rituximab vs. 0/272 (0\%) en el grupo tratado sólo con quimioterapia. En otro RCT, Aviles y cols. ${ }^{32}$, reportaron detalladamente las infecciones ocurridas comparando ciclos de quimioterapia con vs sin rituximab: neumonía $71 / 282(25,2 \%)$ vs $49 / 318(15,4 \%)$, herpes zoster $16 / 282$ $(5,7 \%)$ vs $0 / 318(0 \%)$, infección por CMV 28/282 (9,9\%) vs $3 / 318(0,9 \%)$, infección por virus respiratorio sincicial $7 / 282(2,5 \%)$ vs $0 / 318(0 \%)$ e infección por parvovirus B19 1/282 (0,4\%) vs 0/318 (0\%), respectivamente. La evidencia disponible no permite corroborar la sospecha de un mayor riesgo de reactivación de patógenos latentes en grupos de pacientes seleccionados, como por ejemplo portadores crónicos de hepatitis $\mathrm{B}$.

Rituximab también es utilizado para el tratamiento de AR que no responde a tratamientos tradicionales; pero la evidencia respecto al riesgo de infecciones en este contexto es bastante limitada. En una revisión sistemática de Salliot y cols. ${ }^{33}$, que incluyó tres RCT (1.143 pacientes, seguimiento de 24-48 meses), donde se evaluó el riesgo 
de infección grave con el uso de rituximab a dosis de 500 mg y $1.000 \mathrm{mg}$ vs. placebo, no se evidenció incremento del riesgo: 0/124 (0\%) de infecciones graves con $500 \mathrm{mg}$ de rituximab, 17/621 (2,7\%) con $1.000 \mathrm{mg}$ de rituximab vs $6 / 398(1,5 \%)$ en el grupo placebo. De los 17 casos de infecciones graves con rituximab, la gran mayoría correspondió a infecciones bacterianas ( 5 bronconeumonía, 2 artritis séptica, 3 pielonefritis, 2 gastroenteritis, 1 epiglotitis y 1 celulitis) y sólo una infección viral (hepatitis $\mathrm{B}$ aguda). No hubo pacientes con infecciones oportunistas o TBC. Singh y cols. ${ }^{15}$, tampoco encontraron incremento del riesgo de infecciones graves con el uso de rituximab para el tratamiento de AR: OR 0,75 (IC 95\%; 0,44-1,26), sin describirse detalles respecto a las etiologías de estas infecciones. Suotto y cols. ${ }^{28}$, no evidenciaron incremento en el riesgo de desarrollar una $\mathrm{TBC}$, tanto en $\mathrm{RCT}$, como en estudios de extensión de largo plazo.

\section{Inhibidores de moléculas de adhesión (anti-integrimas): natalizumab y vedolizumab}

Estos anticuerpos monoclonales del tipo anti-integrinas han demostrado su utilidad en el tratamiento de enfermedades inflamatorias intestinales, tanto colitis ulcerosa como enfermedad de Crohn. En una revisión sistemática, Bonobas y cols. ${ }^{26}$, demostraron un incremento no significativo en el riesgo de infecciones oportunistas [TBC, virus JC, nocardiosis, CMV, VEB, candidiasis oral o esofágica, virus varicela-zoster (VVZ), VHS, Pneumocystis jirovecii, Histoplasma capsulatum, neumonía por Legionella u otra infección oportunista no especificada] al comparar el uso de estas moléculas vs placebo, natalizumab: OR 3,49 (IC 95\%; 0,73-16,75) y vedolizumab: OR 0,91 (IC $95 \% ; 0,17-4,90)$. En el sub-análisis para determinar el riesgo de desarrollar una TBC no se logró demostrar mayor riesgo, pero los tamaños muestrales incluidos eran muy pequeños para poder obtener conclusiones objetivas. Al analizar el subgrupo con riesgo de adquirir infecciones oportunistas virales, Wang y cols..$^{34}$, encontraron reporte de éstas en sólo dos RCT (938 pacientes) de natalizumab vs. plabebo, los que consideraban herpes zoster e infección por VHS, sin diferencias significativas entre grupo con natalizumab vs placebo $5 / 474(1,05 \%)$, 4 infecciones por VVZ y 1 por VHS, vs $1 / 464(0,21 \%)$. 1 infección por VHS, $p=0,2$. La misma tendencia se observó con el riesgo de desarrollar influenza 33/474 (7\%) grupo natalizumab vs 20/464 (4,3\%) grupo placebo.

\section{Anticuerpo monoclonal anti-CD52: alemtuzumab}

Es utilizado en pacientes con esclerosis múltiple, leucemia linfática crónica, linfoma no Hodgkin refractarios y leucemia pro-linfocítica de células T. Otras indicaciones son en el rechazo agudo de trasplante de órgano sólido, enfermedad de injerto contra hospedero y en condicionamiento de trasplante, tanto de órgano sólido como de precursores hematopoyéticos. La depleción de linfocitos, (tanto B como T), inducida por alemtuzumab puede ser profunda desde el primer mes de terapia y la reconstitución linfoide puede tardar en promedio, siete meses para linfocitos B, 20 meses para linfocitos T CD8 y hasta 30 meses para linfocitos T CD4 ${ }^{35}$. Skoetz y cols. ${ }^{36}$, en una revisión sistemática que incluyó cinco $\mathrm{RCT}$, compararon la eficacia y efectos adversos de alemtuzumab en el tratamiento de leucemia linfática crónica versus placebo, con rituximab y con quimioterapia convencional. Al considerar los estudios que compararon quimioterapia con vs sin alemtuzumab, hubo un incremento significativo de infecciones entre los pacientes con alemtuzumab RR 1,32 (IC 95\% 1,01-1,74, p = 0,04), con presencia de 7 infecciones graves en la rama alemtuzumab, lo que obligó a la interrupción precoz del estudio. Además, en los mismos estudios se observó una tasa significativamente mayor de reactivación de CMV e infección sintomática por CMV en el grupo de pacientes tratados con alemtuzumab (RR $10,52$, IC $95 \% 1,42-77,68, p=0,02)$, lo que fue observado en ambos estudios. Por otra parte, los estudios en los que se comparó alemtuzumab vs rituximab mostraron mayor ocurrencia de neutropenia febril profunda en los pacientes tratados con alemtuzumab (33 vs $16 \%, p=0,01$ ). Finalmente, en la comparación alemtuzumab vs. quimioterapia con clorambucil se evidenció incremento significativo de infección por CMV, tanto asintomática $(51,7$ vs $7,4 \%)$ como sintomática $(15,4$ vs $0 \%)$. La calidad de los cinco estudios incluidos en esta revisión es considerada como moderada.

En esclerosis múltiple, en cambio, las revisiones sistemáticas disponibles no han evidenciado diferencias significativas en cuanto al riesgo de eventos adversos, incluidas las infecciones ${ }^{37}$. En el estudio CAMMS223 fase 2, que incluyó 334 pacientes sin terapia previa para esclerosis múltiple recidivante activa, recibieron interferón $\beta-1^{\mathrm{a}}$ o alemtuzumab, observando $66 \%$ de infecciones en la rama alemtuzumab vs $47 \%$ en la rama interferón $\beta-1^{\mathrm{a}}$, predominantemente de intensidad leve a moderada, y en el caso de infecciones graves, se presentaron en 4 vs. $2 \%$, respectivamente ${ }^{11}$.

\section{Corolario}

Concluimos, en suma, que efectivamente hay un riesgo incrementado de infecciones asociados al uso de terapias biológicas, pero éstos varían dependiendo por una parte del tipo de biológico utilizado, y por otra parte, del tipo de enfermedad de base para la cual se indica la terapia específica. Por ende, se hace muy necesario el tener 
recomendaciones nacionales de prevención de infecciones en los usuarios de estas terapias que faciliten a los médicos clínicos de distintas especialidades el manejo de las potenciales complicaciones infecciosas derivadas del uso de éstos.

\section{Resumen}

La incorporación de terapias biológicas ha significado un gran avance en el manejo de diversas patologías de origen autoinmune, neoplásico u otros. Si bien su uso ha implicado mejoras significativas en el pronóstico de estas enfermedades, no está exento de complicaciones, entre estas, las infecciosas. El objetivo de este consenso fue evaluar el perfil de seguridad, desde la mirada infectológica, de las terapias biológicas de uso más frecuente y dar recomendaciones para la prevención de infecciones en pacientes tratados con ellas, basándose en la evidencia de mayor calidad disponible para los biológicos seleccionados. El consenso cuenta de dos manuscritos. Esta primera parte detalla los riesgos de desarrollar complicaciones infecciosas dependiendo del tipo de biológico utilizado para determinada patología. La revisión incluyó búsqueda amplia en MEDLINE y Epistemonikos de revisiones sistemáticas y meta-análisis de estudios clínicos controlados y caso/control que examinaban infecciones posteriores al tratamiento con anti-TNF alfa, anti-CD20, anti-CD52, CTLA4-Ig y anti-integrinas. Esta búsqueda se complementó con revisión de cohortes multicéntricas de usuarios de biológicos, del MMWR del CDC, Atlanta, E.U.A. y de registros nacionales y/o de sociedades científicas en la que se hiciera mención a complicaciones infecciosas derivadas del uso de biológicos.

\section{Referencias bibliográfícas}

1.- Waldmann T A. Immunotherapy: past, present and future. Nat Med 2003; 9 (3): 269-77. doi: 10.1038/nm0303-269.

2.- Ruderman EM. Overview of safety of nonbiologic and biologic DMARDs. Rheumatol. 2012; 51 Suppl 6: vi37-43. doi: 10.1093/ rheumatology/kes 283 .

3.- Saag K G, Teng G G, Patkar N M, Anuntiyo J, Finney C, Curtis J R, et al. American College of Rheumatology 2008 recommendations for the use of nonbiologic and biologic diseasemodifying antirheumatic drugs in rheumatoid arthritis. Arthritis Rheum 2008; 59 (6): 762-84. doi: 10.1002/art.23721.

4.- Combe B, Lula S, Boone C, Durez P. Effects of biologic disease-modifying anti-rheumatic drugs on the radiographic progression of rheumatoid arthritis: a systematic literature review. Clin Exp Rheumatol 2018. 36 (4): 65867. https://www.clinexprheumatol.org/article. asp? $\mathrm{a}=11621$.

5.- Papamichael K, Cheifetz AS. Use of anti-TNF drug levels to optimise patient management. Frontline Gastroenterol 2016; 7 (4): 289-300. doi: 10.1136/flgastro-2016-100685.

6.- Rubbert-Roth A, Furst D E, Nebesky J M, Jin A, Berber E. A review of recent advances using tocilizumab in the treatment of rheumatic diseases. Rheumatol Ther 2018; 5 (1): 21-42. doi: 10.1007/s40744-018-0102-x.

7.- Reddy M, Davis C, Wong J, Marsters P, Pendley C, Prabhakar U. Modulation of CLA, IL-12R, CD40L, and IL-2Ralpha expression and inhibition of IL-12- and IL-23-induced cytokine secretion by CNTO 1275. Cell Immunol 2007; 247 (1): 1-11. doi: 10.1016/j. cellimm.2007.06.006.
8.- Kremer J M, Westhovens R, Leon M, Di Giorgio E, Alten R, Steinfeld S, et al. Treatment of rheumatoid arthritis by selective inhibition of T-cell activation with fusion protein CTLA4Ig. New Engl J Med. 2003; 349 (20): 1907-15. doi: 10.1056/NEJMoa035075.

9.- Gurcan H M, Keskin D B, Stern J N, Nitzberg M A, Shekhani H, Ahmed A R. A review of the current use of rituximab in autoimmune diseases. Int Immunopharmacol 2009; 9 (1): 10-25. doi: 10.1016/j.intimp.2008.10.004.

10.- Faissner S, Gold R. Efficacy and safety of the newer multiple sclerosis drugs approved since 2010. CNS Drugs 2018; 32 (3): 269-87. doi: 10.1007/s40263-018-0488-6.

11.- Coles A J, Fox E, Vladic A, Gazda S K, Brinar $\mathrm{V}$, Selmaj K W, et al. Alemtuzumab versus interferon beta-1a in early relapsing-remitting multiple sclerosis: post-hoc and subset analyses of clinical efficacy outcomes. Lancet Neurol 2011; 10 (4): 338-48. doi: 10.1016/S14744422(11)70020-5

12.- Wierda W G, Kipps T J, Keating M J. Novel immune-based treatment strategies for chronic lymphocytic leukemia. J Clin Oncol 2005; 23 (26): 6325-32. doi: 10.1200/JCO.2005.05.008.

13.- Ruck T, Bittner S, Wiendl H, Meuth S G. Alemtuzumab in multiple sclerosis: mechanism of action and beyond. Int J Mol Sci. 2015; 16 (7): 16414-39. doi: 10.3390/ijms160716414.

14.- Singh J A, Wells G A, Christensen R, Tanjong Ghogomu E, Maxwell L, Macdonald J K, et al. Adverse effects of biologics: a network metaanalysis and Cochrane overview. Cochrane Database Syst Rev 2011 (2): CD008794. doi: 10.1002/14651858.CD008794.pub2.

15.- Singh J A, Cameron C, Noorbaloochi S, Cullis T, Tucker M, Christensen R, et al. Risk of serious infection in biological treatment of patients with rheumatoid arthritis: a systematic review and meta-analysis. Lancet 2015; 386 (9990): 258-65. doi: 10.1016/S0140-6736 (14) 61704-9.

16.- Bongartz T, Sutton A J, Sweeting M J, Buchan I, Matteson E L, Montori V. Anti-TNF antibody therapy in rheumatoid arthritis and the risk of serious infections and malignancies: systematic review and meta-analysis of rare harmful effects in randomized controlled trials. JAMA 2006; 295 (19): 2275-85. doi: 10.1001/ jama.295.19.2275.

17.- Keane J, Gershon S, Wise R P, MirabileLevens E, Kasznica J, Schwieterman WD, et al. Tuberculosis associated with infliximab, a tumor necrosis factor alpha-neutralizing agent. New Engl J Med 2001;345 (15): 1098-104. doi: 10.1056/NEJMoa011110.

18.- Gomez-Reino J J, Carmona L, Valverde V R, Mola E M, Montero M D, Group B. Treatment of rheumatoid arthritis with tumor necrosis factor inhibitors may predispose to significant increase in tuberculosis risk: a multicenter active-surveillance report. Arthritis Rheum 2003; 48 (8): 2122-7. doi: 10.1002/art.11137.

19.- Bergstrom L, Yocum D E, Ampel N M, Villanueva I, Lisse J, Gluck O, et al. Increased risk of coccidioidomycosis in patients treated with tumor necrosis factor alpha antagonists. Arthritis Rheum 2004; 50 (6): 1959-66. doi: 10.1002/art.20454

20.- Dixon W G, Hyrich K L, Watson K D, Lunt M, Galloway J, Ustianowski A, et al. Drug-specific risk of tuberculosis in patients with rheumatoid arthritis treated with anti-TNF therapy: results from the British Society for Rheumatology Biologics Register (BSRBR). Ann Rheum Dis 2010; 69 (3): $522-8$. doi: $10.1136 /$ ard.2009.118935. 
21.- Cantini F, Niccoli L, Goletti D. Tuberculosis risk in patients treated with non-anti-tumor necrosis factor-alpha (TNF-alpha) targeted biologics and recently licensed TNF-alpha inhibitors: data from clinical trials and national registries. J Rheumatol Suppl. 2014; 91: 56-64. doi: 10.3899/jrheum.140103.

22.- Winthrop K L, Baxter R, Liu L, Varley C D, Curtis J R, Baddley J W, et al. Mycobacterial diseases and antitumour necrosis factor therapy in USA. Ann Rheum Dis 2013; 72 (1): 37-42. doi: 10.1136/annrheumdis-2011-200690.

23.- Dommasch E D, Abuabara K, Shin D B, Nguyen J, Troxel A B, Gelfand J M. The risk of infection and malignancy with tumor necrosis factor antagonists in adults with psoriatic disease: a systematic review and meta-analysis of randomized controlled trials. J Am Acad Dermatol 2011; 64 (6): 1035-50. doi: 10.1016/j. jaad.2010.09.734.

24.- Yiu Z Z, Exton L S, Jabbar-López Z, Mohd Mustapa M F, Samarasekera E J, Burden A D, et al. Risk of serious infections in patients with psoriasis on biologic therapies: a systematic review and meta-analysis. J Invest Dermatol. 2016; 136 (8): 1584-91. doi: 10.1016/j. jid.2016.03.035.

25.- Ford AC, Peyrin-Biroulet L. Opportunistic infections with anti-tumor necrosis factoralpha therapy in inflammatory bowel disease: meta-analysis of randomized controlled trials. Am J Gastroenterol. 2013; 108 (8): 1268-76. doi: 10.1038/ajg.2013.138

26.- Bonovas S, Fiorino G, Allocca M, Lytras T, Nikolopoulos GK, Peyrin-Biroulet L, et al. Biologic therapies and risk of infection and malignancy in patients with inflammatory bowel disease: a systematic review and network meta-analysis. Clin Gastroenterol Hepatol 2016; 14 (10): 1385-97 e10. doi: 10.1016/j. cgh.2016.04.039.

27.- Strand V, Ahadieh S, French J, Geier J, Krishnaswami S, Menon S, et al. Systematic review and meta-analysis of serious infections with tofacitinib and biologic disease-modifying antirheumatic drug treatment in rheumatoid arthritis clinical trials. Arthritis Res Ther 2015; 17: 362. doi: 10.1186/s13075-015-0880-2.

28.- Souto A, Maneiro J R, Salgado E, Carmona L, Gómez-Reino J J. Risk of tuberculosis in patients with chronic immune-mediated inflammatory diseases treated with biologics and tofacitinib: a systematic review and metaanalysis of randomized controlled trials and long-term extension studies. Rheumatology (Oxford) 2014; 53 (10): 1872-85. doi: 10.1093/ rheumatology/keu172.

29.- Lanini S, Molloy A C, Fine P E, Prentice A G Ippolito G, Kibbler C C. Risk of infection in patients with lymphoma receiving rituximab: systematic review and meta-analysis. BMC Med 2011; 9: 36. doi: 10.1186/1741-7015-9-36.

30.- Coiffier B, Lepage E, Briere J, Herbrecht R, Tilly H, Bouabdallah R, et al. CHOP chemotherapy plus rituximab compared with CHOP alone in elderly patients with diffuse large-B-cell lymphoma. New Engl J Med 2002; 346 (4): 235-42. doi: 10.1056/NEJMoa011795.

31.- Robak T, Dmoszynska A, Solal-Celigny P, Warzocha K, Loscertales J, Catalano $\mathrm{J}$, et al. Rituximab plus fludarabine and cyclophosphamide prolongs progressionfree survival compared with fludarabine and cyclophosphamide alone in previously treated chronic lymphocytic leukemia. J Clin Oncol 2010; 28 (10): 1756-65. doi: 10.1200/ JCO.2009.26.4556.

32.- Aviles A, Neri N, Huerta-Guzmán J, de Jesús Nambo M. ESHAP versus rituximab-ESHAP in frail patients with refractory diffuse large B-cell lymphoma. Clin Lymphoma Myeloma Leuk 2010; 10 (2): 125-8. doi: 10.3816 CLML.2010.n.017.

33.- Salliot C, Dougados M, Gossec L. Risk of serious infections during rituximab, abatacept and anakinra treatments for rheumatoid arthritis: meta-analyses of randomised placebocontrolled trials. Ann Rheum Dis 2009; 68 (1): 25-32. doi: 10.1136/ard.2007.083188.

34.- Wang X, Zhou F, Zhao J, Zhou R, Huang M, Li J, et al. Elevated risk of opportunistic viral infection in patients with Crohn's disease during biological therapies: a meta analysis of randomized controlled trials. Eur J Clin Pharmacol 2013; 69 (11): 1891-9. doi: 10.1007/ s00228-013-1559-8.

35.- Hill-Cawthorne G A, Button T, Tuohy O, Jones J L, May K, Somerfield J, et al. Long term lymphocyte reconstitution after alemtuzumab treatment of multiple sclerosis. J Neurol Neurosurg Psychiatry 2012; 83(3): 298-304. doi: 10.1136/jnnp-2011-300826.

36.- Skoetz N, Bauer K, Elter T, Monsef I, Roloff $\mathrm{V}$, Hallek M, et al. Alemtuzumab for patients with chronic lymphocytic leukaemia. Cochrane Database Syst Rev 2012(2): CD008078. doi: 10.1002/14651858.CD008078.pub2.

37.- Riera R, Porfirio GJ, Torloni MR. Alemtuzumab for multiple sclerosis. Cochrane Database Syst Rev 2016; 4: CD011203. doi: 10.1002/14651858.CD011203.pub2. 